Article

\title{
Tracking Jianghuai Cyclones in China and Their Climate Characteristics
}

\author{
Lan Xia ${ }^{1, *}$ and Yue Zhou ${ }^{2}$ \\ 1 Department of Atmospheric Sciences, Yunnan University, Chenggong University Town, \\ Kunming 650500, China \\ 2 Key Laboratory for Semi-Arid Climate Change of the Ministry of Education, College of Atmospheric \\ Sciences, Lanzhou University, Lanzhou 730000, China; zhouy15@lzu.edu.cn \\ * Correspondence: lanxia@ynu.edu.cn
}

Received: 14 June 2018; Accepted: 26 August 2018; Published: 30 August 2018

check for updates

\begin{abstract}
A Jianghuai cyclone is an extratropical cyclone, which influences the middle and lower reaches of the Yangtze River and Huai River basins in China. According to the definition of Jianghuai cyclones, statistics of their climate characteristics from 1979 to 2010 are obtained by an objective detection and tracking algorithm using ERA-Interim reanalysis data. The results show that the frequency of Jianghuai cyclones has a strong year-to-year variability but no obvious trend. Jianghuai cyclones are most frequent in May but fewest in December. As the cold air is active in spring, which interacts with the warm air from the southwest of the subtropical high at the Yangtze-Huai River region, it makes Jianghuai cyclones occur more frequently in this season. The main origins of Jianghuai cyclones are located in the Poyang Lake region, Dongting Lake region, and Dabie Mountain area. The maximum deepening rate of $0-2 \mathrm{hPa} / 6 \mathrm{~h}$ is featured in $66.4 \%$ of Jianghuai cyclones. Over $40 \%$ of Jianghuai cyclones have a mean deepening rate of $0-1 \mathrm{hPa} / 6 \mathrm{~h}$. The lifetime of Jianghuai cyclones is short, mainly lasting for one to two days. In addition, background characteristics are compared between the formation, climax, and decaying periods of Jianghuai cyclones.
\end{abstract}

Keywords: Jianghuai cyclones; objective detection and tracking algorithm; climatic characteristics

\section{Introduction}

Cyclones and their associated weather are always the focus of public and scientific attention $[1,2]$. A Jianghuai cyclone is an extratropical cyclone, which affects the middle and lower reaches of the Yangtze River and Huai River basins in China. It is one of the most important weather systems related to snow, heavy precipitation, strong winds, and dense fog in the Yangtze-Huai River region of China. There are a few studies by Chinese researchers about case studies of Jianghuai cyclones. For example, $\mathrm{Xu}$ and Jiao [3] analyzed two cases of Jianghuai cyclones and described their distributions of pressure, divergence, vorticity, and velocity. Zhang and Wang [4] used 13 cases of Jianghuai cyclones to perform a synthesis analysis. They found that for the large-scale conditions and structures of $12 \mathrm{~h}$ after the formation of Jianghuai cyclones, the lower atmospheric levels (surface, $850 \mathrm{hPa}$, and $700 \mathrm{hPa}$ ) of Jianghuai cyclones are characterized by low pressures associated with airflow convergence, while higher levels $(400 \mathrm{hPa}, 300 \mathrm{hPa}$, and $200 \mathrm{hPa})$ feature high pressures with divergence. Hou [5] studied the structures of two cases of summer Jianghuai cyclones. One case presented a baroclinic structure and the other one showed an equivalent barotropic structure. Wei et al. [6] used the historical weather charts from the National Meteorological Centre of China to analyze the track, origin, and frequency of Jianghuai cyclones. Wang et al. [7] showed the climactic characteristics and inter-annual variations of Jianghuai cyclones in late spring and early summer from 1980 to 2012. 
However, the study of Jianghuai cyclones is still deficient and mainly concentrates on case studies. It is essential to know the climatic characteristics of Jianghuai cyclones. That is the aim of this study.

An automatic detection and tracking algorithm [8-13] provide the possibility to track cyclones in an efficient and objective way. This technique allows for analyzing long-term climatologies of cyclones. For example, Xia et al. $[14,15]$ studied quasi-millennium climatologies of extratropical cyclones of the northern and southern hemispheres within climate model simulations. We here apply the detection and tracking method to Jianghuai cyclones to record their tracks, core pressures, lifetime, and other related fields.

This paper is organized as follows: the data and tracking methods are introduced in Section 2. Case studies of Jianghuai cyclones and comparisons of reanalysis tracking results with observations are shown in Section 3.1. Climatic characteristics of Jianghuai cyclones are given in Section 3.2. Large-scale background fields are compared between the genesis, climax, and lysis periods of Jianghuai cyclones in Section 3.3. The summary is presented in Section 4.

\section{Data and Tracking Method}

\subsection{Data}

In this paper, we analyzed mean sea level pressure (MSLP), air temperature, vertical integral of water vapor, horizontal winds, geopotential height, and divergence of the ERA-Interim dataset [16] by the European Centre for Medium-Range Weather Forecasts (ECMWF) for the period 1979-2010. The temporal resolution was 6 hours and the spatial resolution was $1^{\circ} \times 1^{\circ}$. More technical details of this dataset can be found in Berrisford et al. [17].

The application and validation of ERA-Interim in China has been investigated $[18,19]$. Gao et al. [18] compared the monthly temperature between 756 Chinese observation stations and ERA-Interim and found that ERA-Interim reanalysis can reflect the inter-annual variability of observations very well. Gao et al. [19] assessed the air temperature, pressure, and sea surface temperature (SST) of ERA-Interim from 1979 to 2012 by comparing with Chinese costal observation stations. They found that the characteristics of the seasonal changes of ERA-Interim agree well with the observations. The aforementioned studies show that the ERA-Interim reanalysis provide realistic data, which can be used to analyze China's climatology.

\subsection{Detection and Tracking Method}

According to the Jiangsu Meteorological Bureau [20], the definition of a Jianghuai cyclone is a low-pressure system which originates in the middle and lower reaches of the Yangtze-Huai River basin in China with a lifetime longer than $12 \mathrm{~h}$ and which features a front structure. Figure 1 shows the geographical regions affected by Jianghuai cyclones $[6,20]$. When the cyclone originates in the box of Figure 1, it can be considered as a possible Jianghuai cyclone.

Firstly, MSLP minima were recorded as potential centers of Jianghuai cyclones by comparing with the surrounding grids. To remove weak low-pressure systems, a MSLP gradient of more than $0.6 \mathrm{hPa}$ was requested in a distance of $1^{\circ}$ (approximately $100 \mathrm{~km}$ ) from the minimum. As Jianghuai cyclones usually accompany front structures, a minimum temperature gradient on $925 \mathrm{hPa}$ of over $3.1 \mathrm{~K} / 100 \mathrm{~km}$ should be fulfilled. MSLP minima, which satisfy these requirements, were retained as the positions of Jianghuai cyclones. Secondly, cyclone positions were combined as tracks of Jianghuai cyclones. The distance between two time steps should be less than $500 \mathrm{~km}$ [21]. The tracks should last at least $12 \mathrm{~h}$ (i.e., two points). More details and information on the reliability of the detection and tracking algorithm can be found in Zahn et al. [13] and Xia et al. [22]. 


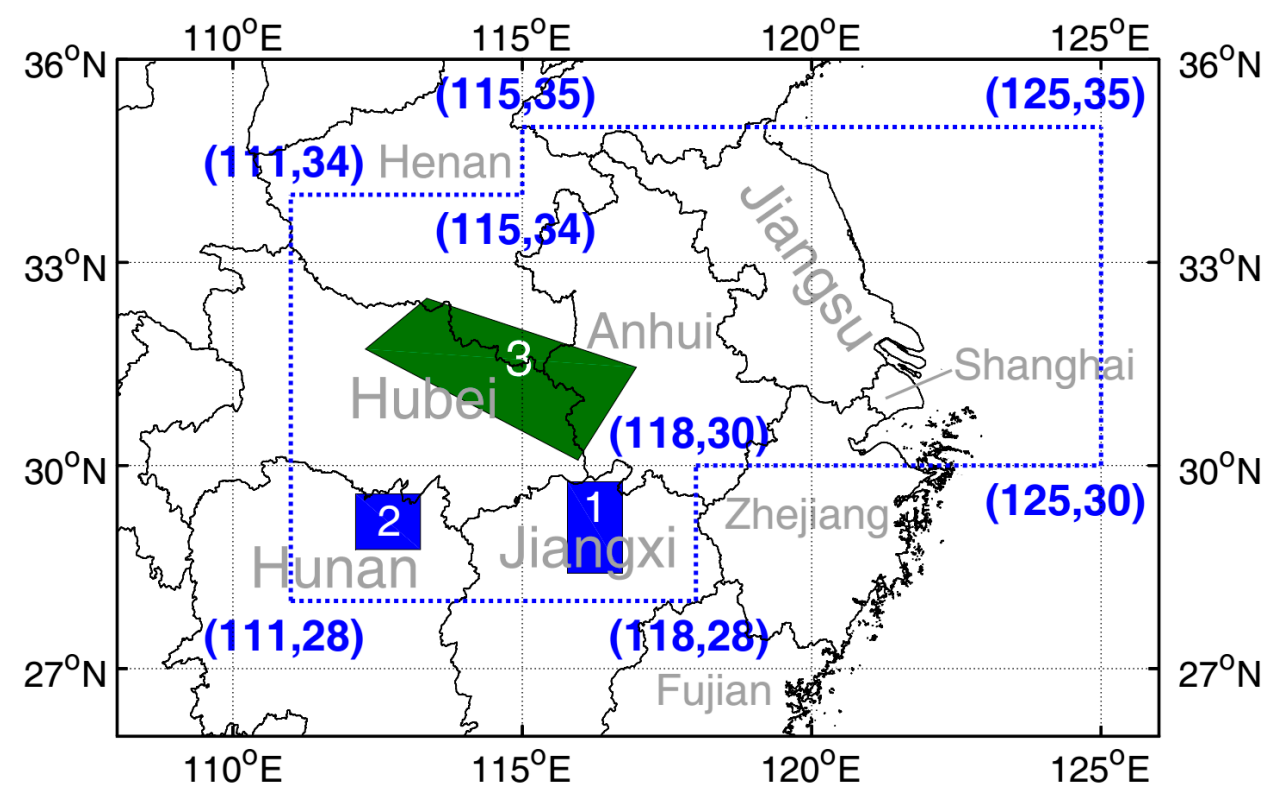

Figure 1. The defined region of Jianghuai cyclones as an irregular rectangle [6,20]. Numbers in brackets provide longitudes and latitudes of the irregular rectangle. Provinces or city in Yangtze-Huai River basin are given in gray text. Major lakes and mountain areas are exhibited as colored patches and numbers. Poyang Lake (No. 1 blue patch) and Dongting Lake (No. 2 blue patch) are the largest and second largest freshwater lake in China, respectively. Dabie Mountain is the watershed of Yangtze River and Huai River (No. 3 green patch).

\section{Results}

\subsection{Tracking Jianghuai Cyclones}

Jianghuai cyclones from 1979 to 2010 (32 years) are tracked using the detection and tracking algorithm described in Section 2. To make sure the statistics of Jianghuai cyclones obtained from the tracking algorithm are reliable, cyclone numbers and genesis times are compared with observation data. The Weather Bureau of Jiangsu Province has reported records of Jianghuai cyclones from 1961 to 1980 [20]. Unfortunately, this data only contains the numbers and genesis times of Jianghuai cyclones each year and not the entire track information. The comparison investigates numbers and genesis times in 1979 and 1980, the results are shown in Table 1. In 1979, there were 17 Jianghuai cyclones in the observation records. Seventeen Jianghuai cyclones are also tracked in the ERA-Interim reanalysis data by the tracking algorithm in 1979 . The genesis times of 13 cyclones $(76.5 \%)$ agree well with the observation records. In 1980, 15 Jianghuai cyclones were tracked in the reanalysis and as well as in the observation records and 9 of them $(60 \%)$ coincide with genesis times. We can see that the numbers of Jianghuai cyclones detected by the tracking algorithm have good consistency with the observation records, although the genesis times have some differences. More important is that the climatological statistics obtained by the tracking algorithm is in accordance with the observation records. It is noted that historical records depend on judgments of weather charts by forecasters. It might be subjective and may lead to uncertainty. It is strongly argued that statistics of Jianghuai cyclones obtained by an objective detection and tracking algorithm can keep good homogeneity over time, which is more favorable for statistics of climatology analysis.

In Figure 2, the tracks of two Jianghuai cyclones in ERA-Interim derived by the tracking algorithm (orange lines) are compared with the observations (blue lines). The two cases are from Sun et al. [23]. One cyclone generated in 8 April 2008 and moved eastward, and the other started in 19 April 2009 and took a northeastward moving path (Figure 2: blue lines). Upper numbers of Figure 2 are observed 
occurrence times of cyclones, for example, 0820 means 20:00 China Standard Time (CST, the same below) on 8 April [23]. Lower numbers of Figure 2 are measured minima cyclone pressures (unit: hPa).

Table 1. Comparisons of Jianghuai cyclones between the observations and reanalysis data derived by the tracking method. The observations are records of Jianghuai cyclones by the Weather Bureau of Jiangsu Province [20]. Numbers and genesis times of Jianghuai cyclones derived from ERA-Interim by the tracking method are compared with observations. Overlapping cyclones are defined when genesis times of cyclones in the reanalysis data by the tracking method coincide exactly with genesis times in the observation records.

\begin{tabular}{ccccc}
\hline Year & $\begin{array}{c}\text { Numbers of } \\
\text { Observation }\end{array}$ & $\begin{array}{c}\text { Numbers by } \\
\text { Tracking Method }\end{array}$ & $\begin{array}{c}\text { Overlapping } \\
\text { Cyclones }\end{array}$ & $\begin{array}{c}\text { Percentage of } \\
\text { Overlapping }\end{array}$ \\
\hline 1979 & 17 & 17 & 13 & $76.5 \%$ \\
1980 & 15 & 15 & 9 & $60.0 \%$ \\
\hline
\end{tabular}

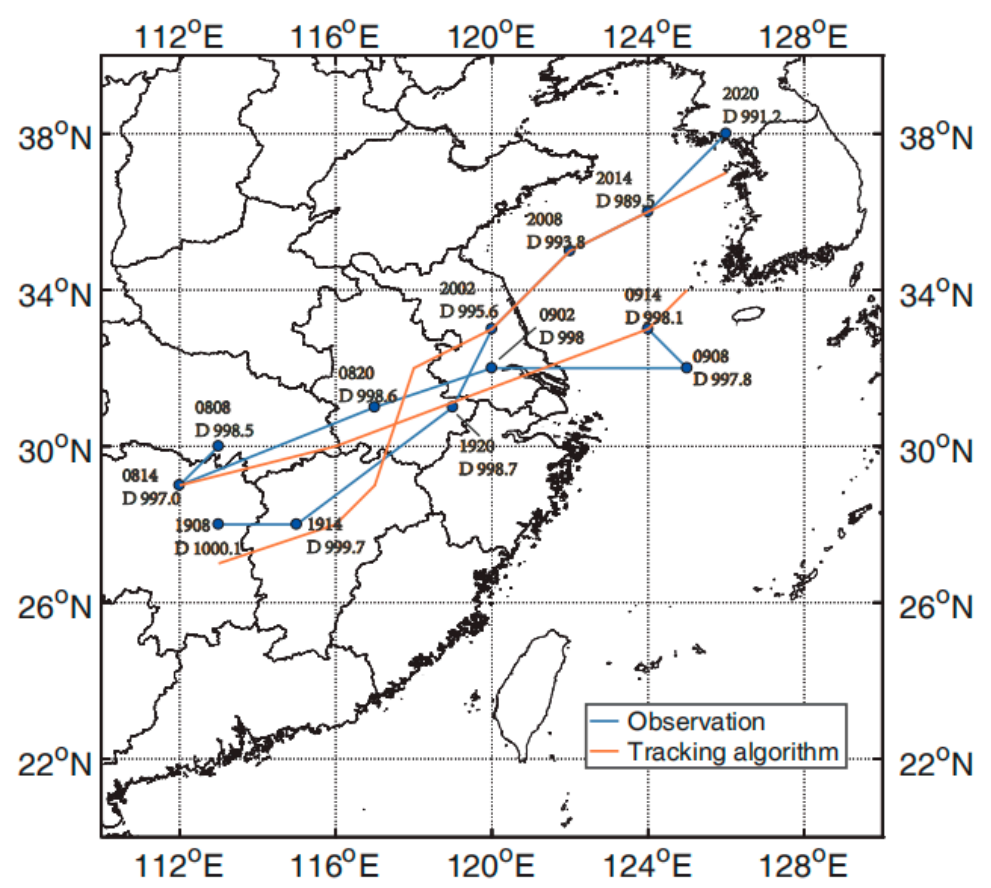

Figure 2. The tracks of two Jianghuai cyclones. The blue lines are observed tracks from Sun et al. [23] and the orange lines are tracks from ERA-Interim derived by the tracking algorithm. The upper number is the observation time, for example 2008 means 08:00 CST (China Standard Time) on 20 April. The lower number is the minimum pressure $(\mathrm{hPa})$ of the cyclone center and ' $\mathrm{D}$ ' represents low-pressure systems [23].

The first case is the Jianghuai cyclone associated with a stationary front [23]. It generated on 8 April 2008. The moving path is west-east way. After 02:00 9 April, it moved over the sea and regenerated with stronger intensity. The tracking algorithm detected the cyclone at 02:00 8 April and its decay at 08:00 9 April in the reanalysis data. Both times are one time step ahead of the observations, but the durations of the cyclone are the same. Generally speaking, the path obtained from the tracking algorithm agrees well with the observed one.

The second case was formed by an inverted trough [23]. It started at 08:00, 19 April 2009 (Figure 2) and centered at $113^{\circ} \mathrm{E}, 28^{\circ} \mathrm{N}$. It moved northeastward and strengthened. After 02:00 on 20 April the cyclone entered the sea and its intensity became stronger over the sea. On 20 April at 20:00, the cyclone reached the Korean Peninsula and dissipated after moving northeastward. The detection and tracking 
method tracked this cyclone in the reanalysis data beginning at $02: 00$ on 19 April 2009 at $113^{\circ} \mathrm{E}, 27^{\circ} \mathrm{N}$. The time of origin is a little earlier and the position is slightly southward. The position of the cyclone at 20:00 on 19 April in the reanalysis data has deviations from the observation record in that the position derived from the tracking method is a little northwest. The tracking paths of both reanalysis data and observations coincide with each other well afterward and the dissipation time is exactly the same.

We can see that the paths of Jianghuai cyclones derived from ERA-Interim by the tracking algorithm are in good agreement with the observations. At the same time, numbers and times of Jianghuai cyclones obtained by the algorithm are in accordance with historical records. We therefore conclude that the statistics and tracks of Jianghuai cyclones derived with the tracking algorithm are reliable and can be used to investigate the climatic characteristics of Jianghuai cyclones.

\subsection{Climatological Characteristics of Jianghuai Cyclones}

There are 452 Jianghuai cyclones in total and on average 14.125 cyclones per year in the ERA-Interim reanalysis data. This result is in agreement with the conclusions obtained by Wei et al. [6]. The blue line of Figure 3 shows the annual numbers of Jianghuai cyclones. Jianghuai cyclones are most frequent with a value of 19 in 1983, 2003, and 2006. The minimum number of Jianghuai cyclones occurs in 1988, only seven Jianghuai cyclones formed. Jianghuai cyclones are also much fewer in number in 1984, with nine occurrences. We can see an obvious inter-annual variability in the number of Jianghuai cyclones. Figure 3 also shows the 10-year running average of the numbers of Jianghuai cyclones (orange line). The 10-year moving average frequency of Jianghuai cyclones shows no large differences from 1985 to 2000 . However, after 2000 there is a slightly increasing trend, but this trend is not significant. This is accordance with the results of Wang et al. [7]. Qin et al. [24] analyzed the characteristics of cyclones entering coastal waters of China and found that the numbers of cyclones increased insignificantly from 1979 to 2012. This finding also supports our results.

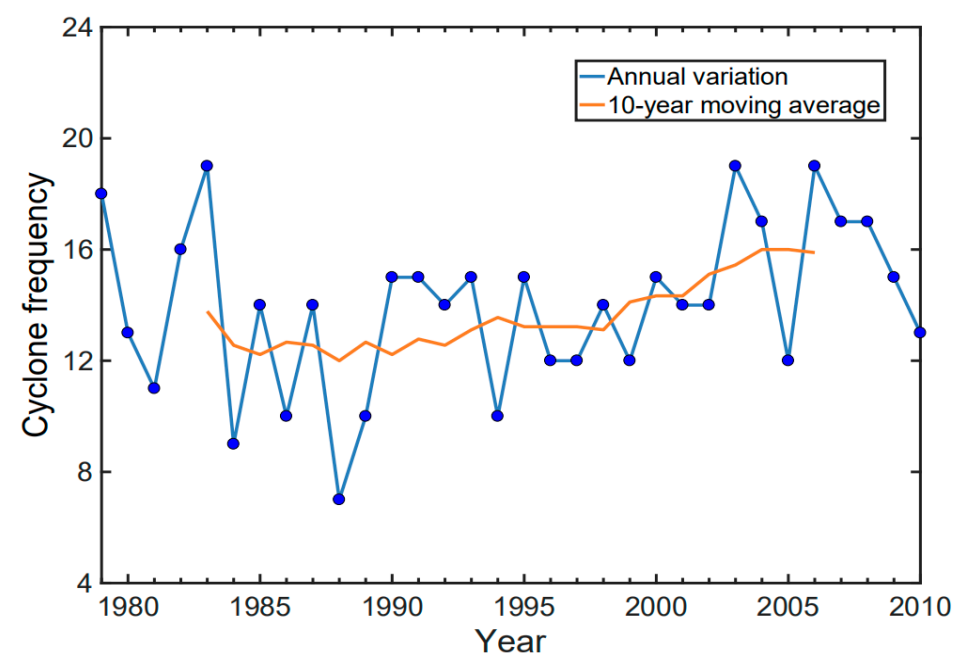

Figure 3. Blue line: annual variability of the numbers of Jianghuai cyclones from 1979 to 2010 derived from ERA-Interim reanalysis; orange line: the 10-year moving average of the numbers of Jianghuai cyclones from 1979 to 2010.

Figure 4 shows the monthly numbers of Jianghuai cyclones. It can be seen that Jianghuai cyclones occur most often in April, May, and June. The numbers of these three months account for $43.8 \%$ of the total number of Jianghuai cyclones. Jianghuai cyclones occur most often in May with 67 events (Figure 4). The lowest number is in December (Figure 4: 16), only accounting for $3.5 \%$ of the total number. These results agree well with the conclusions of Liu et al. [25]. Table 2 shows the seasonal distribution of Jianghuai cyclones based on ERA-Interim reanalysis data. Jianghuai cyclones are active in spring and summer, which accounts for $38.5 \%$ and $29.2 \%$ of the total number, respectively. This is 
consistent with the monthly distribution of Jianghuai cyclones. In spring, the cold air is still actively encountering the moist southwesterly airflow from the northern side of the Western Pacific subtropical high. It provides the favorable baroclinic conditions for forming Jianghuai cyclones. In summer, warm temperatures and adequate moisture also support the generation and development of Jianghuai cyclones, especially in the plum rain season in Southeastern China. However, in winter, East Asia is controlled by the Mongolia and Siberia cold high, which is strong and stable. The Jianghuai cyclone activity is quite weak and is lowest in winter.

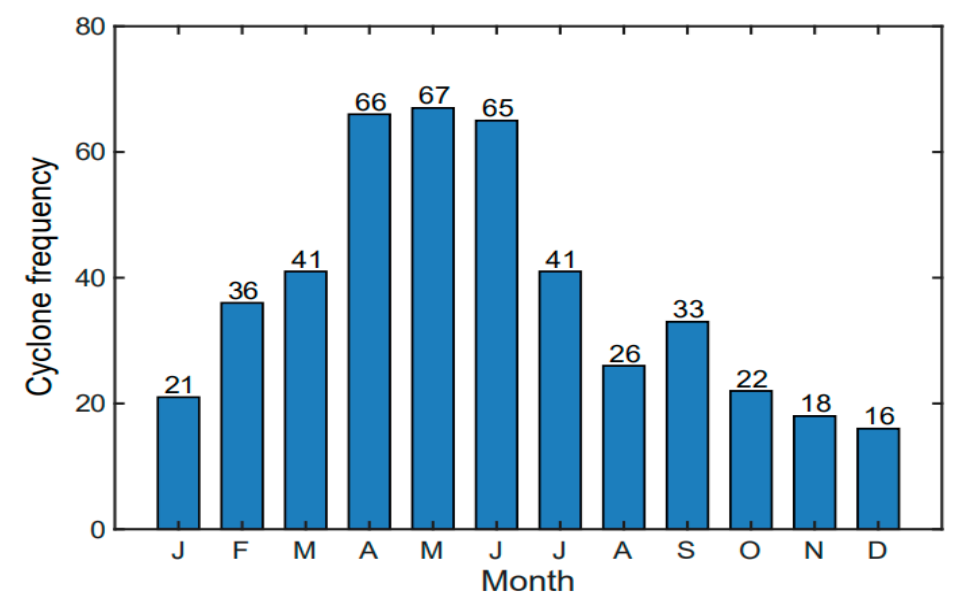

Figure 4. The monthly numbers of Jianghuai cyclones derived from ERA-Interim reanalysis by the tracking algorithm.

Table 2. The seasonal distributions (\%) of the numbers of Jianghuai cyclones derived from ERA-Interim. Spring is defined as March, April, and May. Summer is June, July, and August. Autumn is September, October, and November. Winter is January, February, and December.

\begin{tabular}{cccccc}
\hline Season & Spring & Summer & Autumn & Winter & Total \\
\hline number & 174 & 132 & 73 & 73 & 452 \\
percentage & $38.5 \%$ & $29.2 \%$ & $16.2 \%$ & $16.2 \%$ & $100 \%$ \\
\hline
\end{tabular}

Figure 5a shows the regional density of Jianghuai cyclones. The Jianghuai cyclones mainly influence the Dongting Lake area in Hunan Province, most regions of Jiangxi Province, and the Jiangsu-Anhui plain. The areas influenced by Jianghuai cyclones include the lower reaches of the Yangtze River and the Huai River, such as Zhejiang Province and the north of Fujian Province. In Figure 5b, we can see the origin area of Jianghuai cyclones. It has been found that the Jianghuai cyclones generate in the Dongting Lake area, Poyang Lake area, and the northeast side of Dabie Mountain area. This is in agreement with the results of Wei et al. [6]. As the Poyang and Dongting lakes are the largest and second largest freshwater lakes in China, the warm surface temperature and abundant water vapor over the lakes provide favorable conditions for the formation and development of Jianghuai cyclones. According to the conservation of potential vorticity, it is easy to generate low-pressure systems at the lee sides of the mountains [26], which leads to another high-frequency origin area of Jianghuai cyclones. Figure $5 c$ gives the distributions of lysis areas of Jianghuai cyclones. The maxima are located in the northwest of Fujian Province, that is, south of the Wuyi Mountains. Jianghuai cyclones might be prompted to decay after crossing the Wuyi Mountains, then be blocked by mountain areas in the northeast of Fujian and vanish there. Other main lysis areas can be found in the northeast of Hunan and Jiangxi, the south of Jiangxi, the middle of Zhejiang, and along the coastlines. These places are downstream regions corresponding to the genesis areas. Jianghuai cyclones can reach to the Yellow Sea of China, and as far as the Korean Peninsula and Japan. 

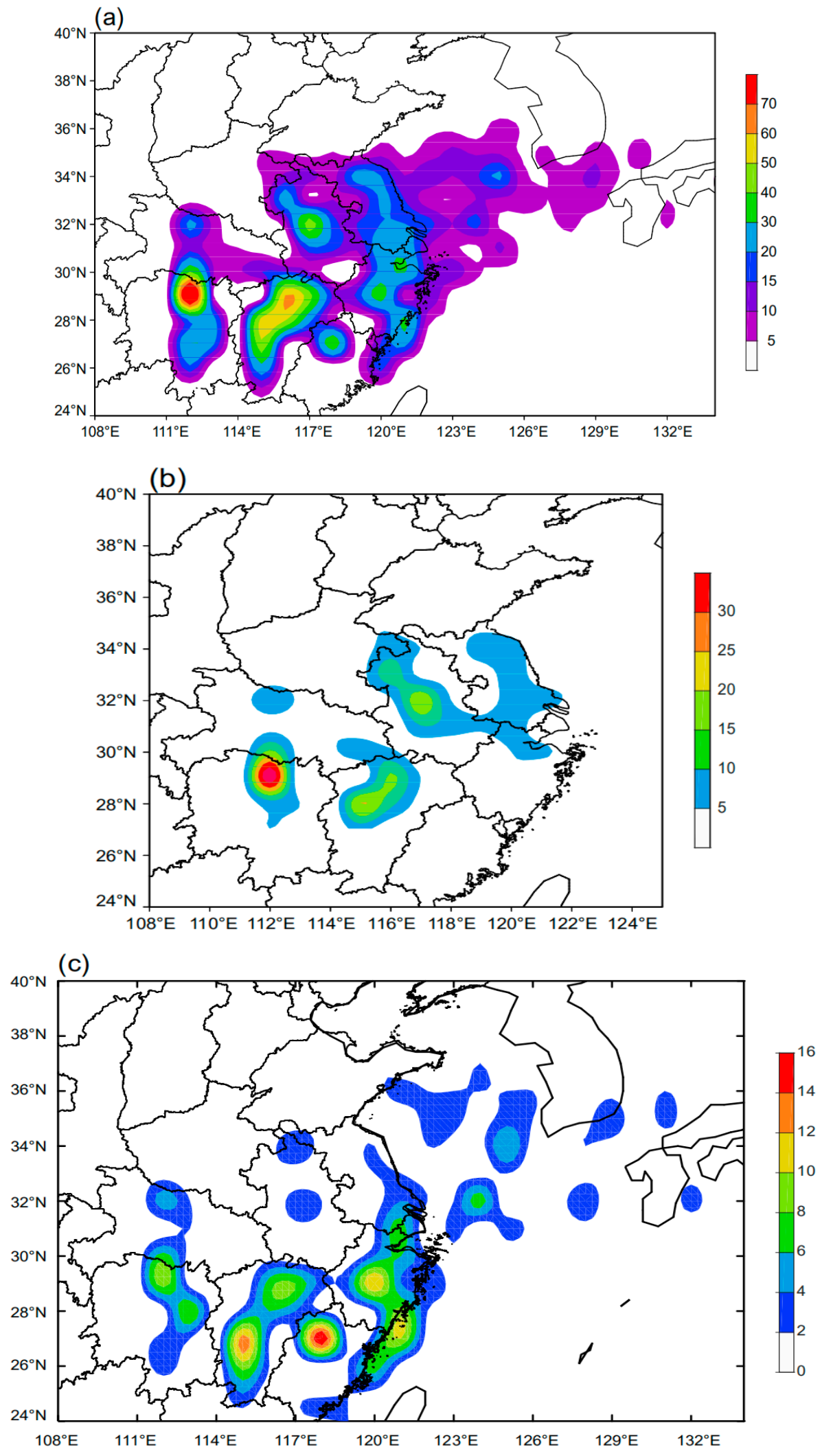

Figure 5. (a) The spatial density (cyclones $/ 10,000 \mathrm{~km}^{2}$ ), (b) the origin density (cyclones $/ 10,000 \mathrm{~km}^{2}$ ) and (c) the lysis density (cyclones $/ 10,000 \mathrm{~km}^{2}$ ) of Jianghuai cyclones derived from ERA-Interim reanalysis. The corresponding geographic information can be found in Figure 2. 
Figure 6 shows the lifetime distribution of Jianghuai cyclones. The most common lifetime of Jianghuai cyclones lasts one to two days, which accounts for $49.06 \%$ of all cyclones. The percentage of Jianghuai cyclones lasting less than one day is $36.2 \%$. Most of the Jianghuai cyclones survive less than three days $(98.34 \%)$. As Jianghuai cyclones mainly travel on the land, it might be not conducive to the cyclone surviving. On the other hand, it could be possible that at the time of the generation and dissipation, Jianghuai cyclones are too weak to be detected and tracked, which may lead to the detected short life history of Jianghuai cyclones.

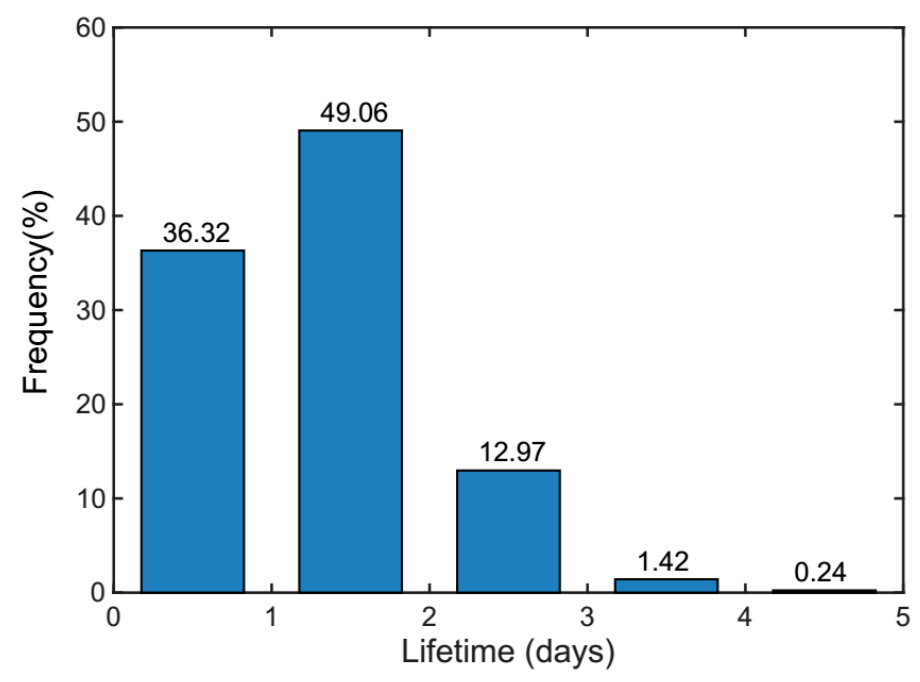

Figure 6. The distributions (\%) of Jianghuai cyclone lifetime (days) derived from ERA-Interim analysis.

Figure 7a shows the distributions of the average deepening rates of Jianghuai cyclone pressures per $6 \mathrm{~h}$. It is obvious that the decreases of central pressure of Jianghuai cyclones are mainly between 0 and $1 \mathrm{hPa}$ per $6 \mathrm{~h}$, accounting for $43.87 \%$ of the total dataset. A deepening rate between 1 and $2 \mathrm{hPa} / 6 \mathrm{~h}$ is shown in $39.03 \%$ of all Jianghuai cyclones. The cyclone-average deepening pressure of Jianghuai cyclones is mostly between 0 and $4 \mathrm{hPa}$ per $6 \mathrm{~h}$, which occurs for $97.73 \%$ of the total number. Jianghuai cyclones that show a core pressure deepening rate of over $4 \mathrm{hPa}$ per $6 \mathrm{~h}$ are very rare (only $2.27 \%$ ). Therefore, the strength of a Jianghuai cyclone is generally not very strong. Figure $7 \mathrm{~b}$ shows the maximum core pressure deepening rates per $6 \mathrm{~h}$ of Jianghuai cyclones. If the central pressure of a cyclone decreases sharply in a short time, it will be an explosive cyclone, which is potentially dangerous with heavier rain and stronger wind speeds [27]. We find that the maximum deepening rate of Jianghuai cyclones lies most often between 0 and $2 \mathrm{hPa} / 6 \mathrm{~h}(66.38 \%)$. The second highest maximum deepening rate can be found between 2 and $4 \mathrm{hPa} / 6 \mathrm{~h}$ with $25.93 \%$ of all cases. A maximum deepening pressure between 0 and $6 \mathrm{hPa} / 6 \mathrm{~h}$ is found in $97.15 \%$ of all Jianghuai cyclones. There are only a few Jianghuai cyclones $(2.85 \%)$ with a maximum deepening pressure larger than $6 \mathrm{hPa} / 6 \mathrm{~h}$. Usually marine cyclones grow explosively due to the abundant water vapor and latent heat release over the sea, which increases the baroclinicity [28]. However, Jianghuai cyclones develop on the land most of the time, which is not conducive for explosive growth. Generally, Jianghuai cyclones are not so powerful. This could also explain why Jianghuai cyclones cannot survive very long time spans, as shown before (Figure 6).

The initial pressures of Jianghuai cyclones were analyzed for different seasons (Table 3) and months (Figure 8). The lower the initial central pressure is, the stronger is the formation of a Jianghuai cyclone. The formation of a Jianghuai cyclone is the strongest in summer (July), while it is the weakest in winter (December), as shown in Table 3 and Figure 8. Again, due to the large amount of released latent heat and abundant water vapor in the plum rain season, the initial intensity of Jianghuai cyclones is relatively stronger in summer. It should be noted that spatial filtering which can remove 
the large-scale systems $[29,30]$ is not applied before tracking in this study. Although we applied the criteria of gradients for detection, large-scale background fields can still possibly influence cyclone pressures, especially in the initial period.
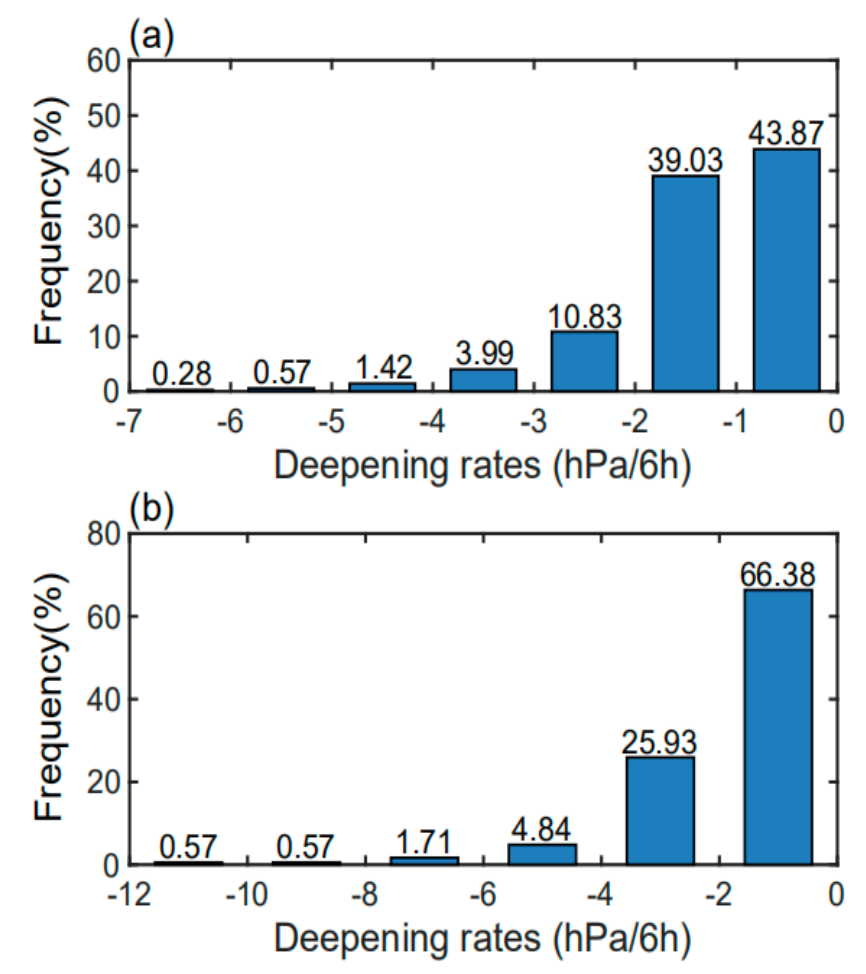

Figure 7. Distributions (\%) of (a) the average deepening rates (hPa/6h); (b) the maximum deepening rates $(\mathrm{hPa} / 6 \mathrm{~h})$ of Jianghuai cyclones derived from ERA-Interim reanalysis.

Table 3. The seasonal changes of average origin central pressures $(\mathrm{hPa})$ of Jianghuai cyclones derived from ERA-Interim reanalysis. Seasons are defined the same as in Table 2.

\begin{tabular}{ccccc}
\hline Season & Spring & Summer & Autumn & Winter \\
\hline Initial pressure $(\mathrm{hPa})$ & 1006.4 & 1002.8 & 1011.2 & 1016.8 \\
\hline
\end{tabular}

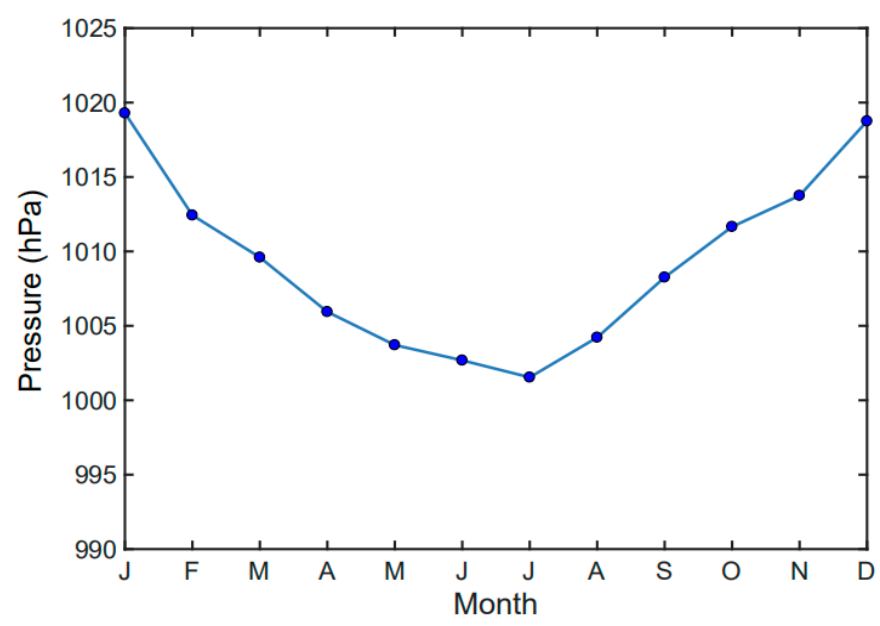

Figure 8. The average initial pressures $(\mathrm{hPa})$ of Jianghuai cyclones over different months derived from ERA-Interim reanalysis. 


\subsection{Background Characteristics in the Genesis, Climax, and Lysis Periods of Jianghuai Cyclones}

The mean fields at the genesis, climax, and lysis times of Jianghuai cyclones are examined to investigate the large-scale background characteristics. The genesis (lysis) time is the first (last) time in the lifespan of a Jianghuai cyclone. The climax time is chosen as the time of the lowest cyclone MSLP attained. The background fields, including vertical integral of water vapor, MSLP, horizontal divergence, geopotential height, and horizontal wind field, are studied by averaging in the genesis, climax, and lysis times of all Jianghuai cyclones.

From Figure 9a, we can find that in the genesis period, a wet tongue spreads from the mouth of the Yangtze River to the Jiangsu, Anhui, Hunan, and Jiangxi Provinces. The highest water vapor centers correspond to the lake regions, as well as the genesis areas of Jianghuai cyclones. It should be noted that the water vapor around Fujian and south of Zhejiang Provinces near the sea is relatively lower. Jianghuai cyclones can form with sufficient water vapor. However, when Jianghuai cyclones move out from the lake region, the water vapor supply reduces and might be not favorable for sustaining them until they move into the sea. This could explain why the lifetime of a Jianghuai cyclone is not very long. The wet tongue weakens and retreats to the mouth of the Yangtze River (Figure 9b) while Jianghuai cyclones develop. Water vapor maxima over the lake regions decrease compared with the genesis time. At the lysis time (Figure 9c), the wet tongue disappears over the land and water vapor maxima of Hunan and Jiangxi subdue so much that it is unfavorable for Jianghuai cyclones to survive.
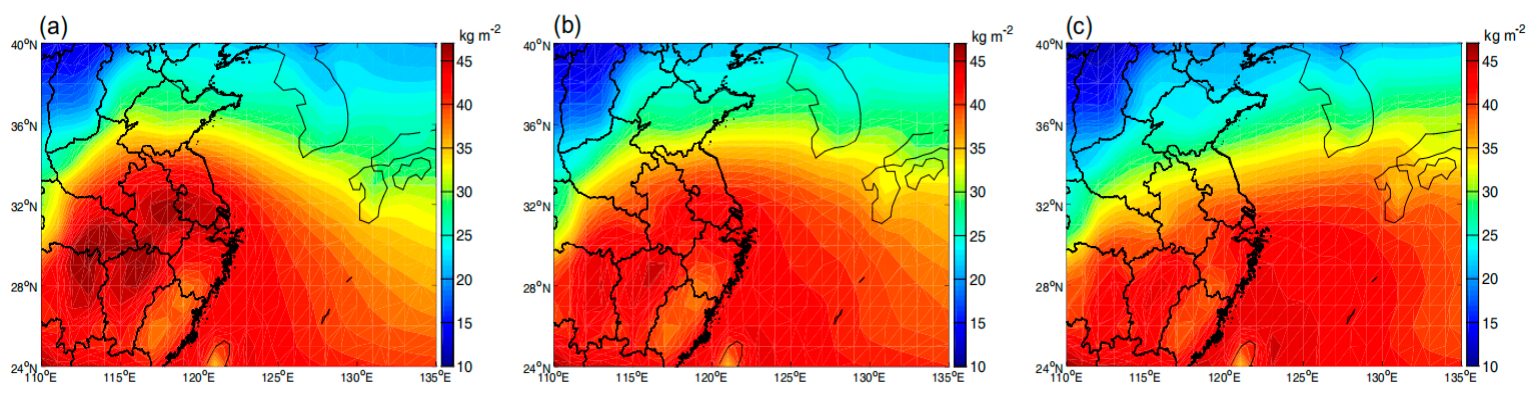

Figure 9. The average vertical integral of water vapor $\left(\mathrm{kg} / \mathrm{m}^{2}\right)$ at $(\mathbf{a})$ the genesis time, (b) the climax time, and (c) the lysis time of Jianghuai cyclones from 1979 to 2010 derived from ERA-Interim.

At the genesis time, MSLP is lower around the Yangtze-Huai River basin (Figure 10a). At the same time, convergence can be found over this area at $850 \mathrm{hPa}$ (Figure 11a) while divergence occurs over the Yangtze-Huai River basins at $200 \mathrm{hPa}$ (Figure 11d). It indicates that the ascending flow is strong over this region. However, the center of divergence is located northeast over the Yellow Sea. Lower heights lie in the southeast of China at $850 \mathrm{hPa}$ (Figure 12a), which is opposite at $500 \mathrm{hPa}$ and $200 \mathrm{hPa}$ levels (not shown), that is, higher heights are located in these areas. The lowest geopotential height center appears around the boundary between Shandong and Anhui Province (Figure 12a). Cyclonic winds are corresponding to this height low (Figure 12a). The trough line of geopotential height extends southwestward. The MSLP low is situated at the front of the height trough at $850 \mathrm{hPa}$. North winds (cold air) leading around the trough at $850 \mathrm{hPa}$ could meet with the southwest winds (warm and humid air) over the Yangtze-Huai River basin (Figure 12a) which increase the baroclinicity. Higher humidity, lower MSLP, and ascending air accompanied with the intersections of north and south winds all provide favorable backgrounds for formations of Jianghuai cyclones. 

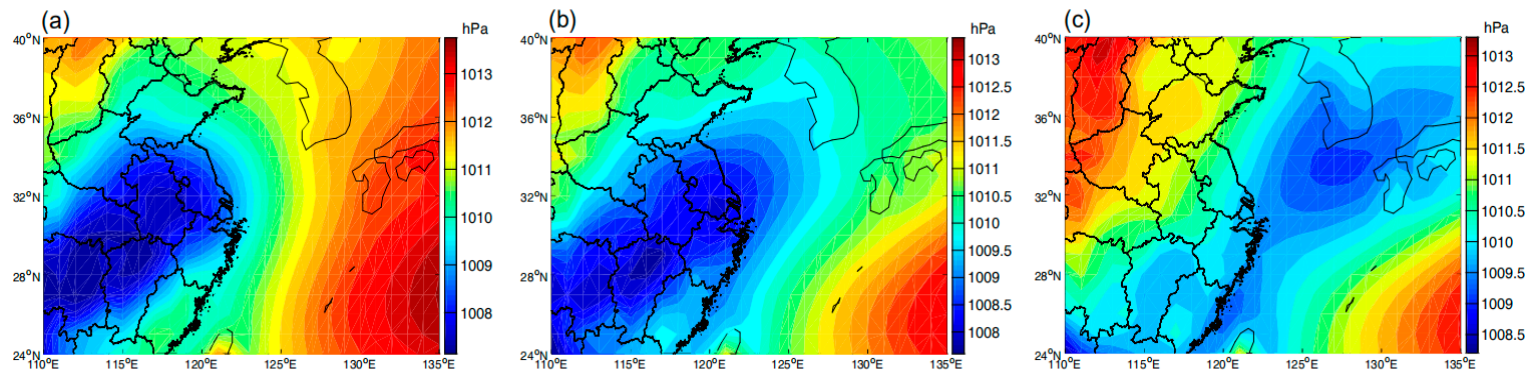

Figure 10. The mean sea level pressure ( $\mathrm{hPa}$ ) at (a) the genesis time, (b) the climax time, and (c) the lysis time of Jianghuai cyclones from 1979 to 2010 derived from ERA-Interim.
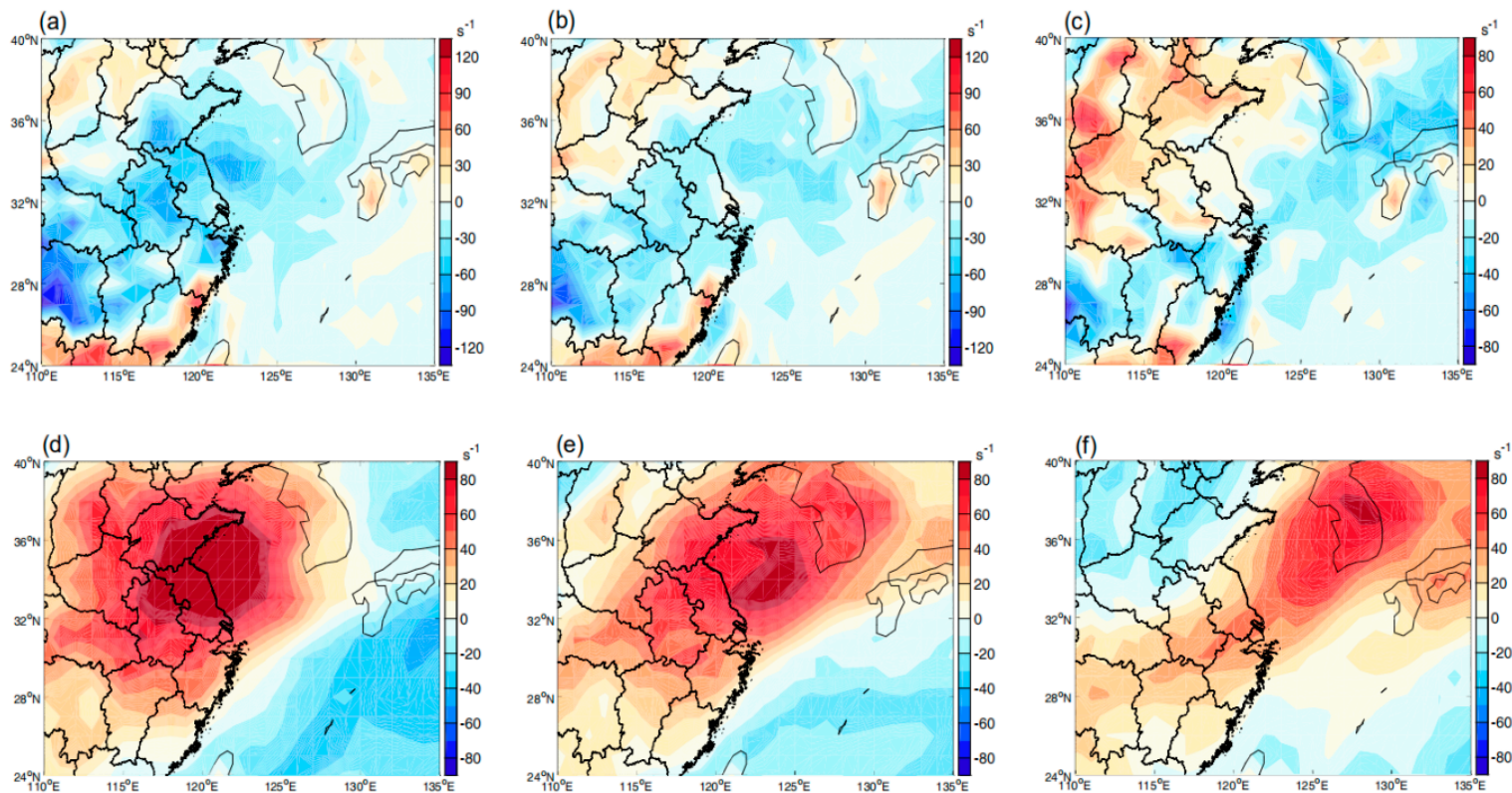

Figure 11. The average horizontal divergence $\left(\mathrm{s}^{-1}\right)$ at $850 \mathrm{hPa}$ in (a) the genesis time, $(\mathbf{b})$ the climax time, and (c) the lysis time of Jianghuai cyclones from 1979 to 2010. Panels (d,e,f) are the same, but for the average horizontal divergence $\left(\mathrm{s}^{-1}\right)$ at $200 \mathrm{hPa}$.
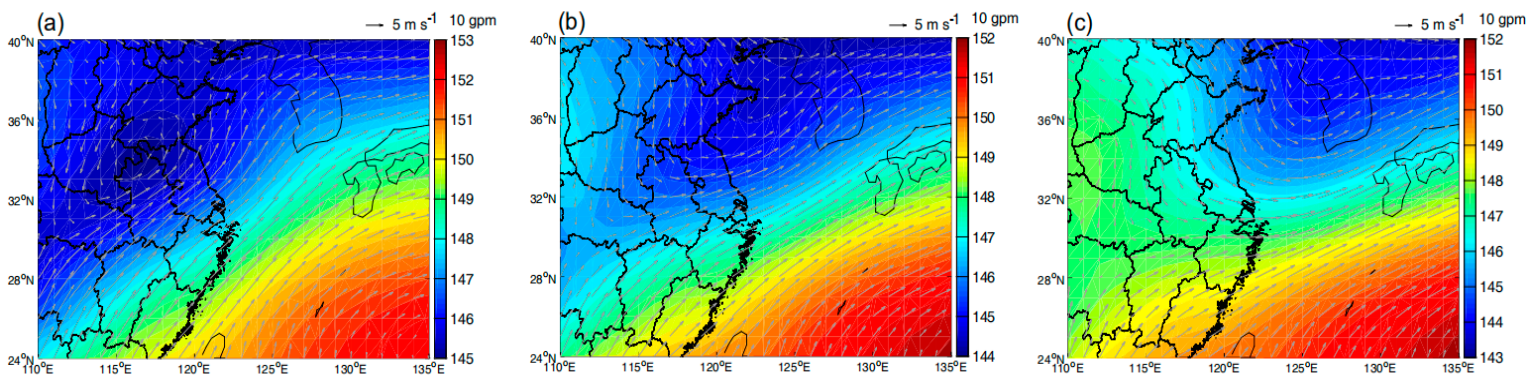

Figure 12. The average geopotential height (gpm, shown as filled colors) and horizontal wind field ( $\mathrm{m} \mathrm{s}^{-1}$, shown as gray arrows) at $850 \mathrm{hPa}$ in (a) the genesis time, (b) the climax time, and (c) the lysis time of Jianghuai cyclones from 1979 to 2010. 
At the climax time, which is defined as MSLP of a Jianghuai cyclone deepens to the minimum, the average vertical integral of water vapor already begins to reduce (Figure 9b). MSLP lows enlarge to the Yellow Sea (Figure 10b). However, convergence at $850 \mathrm{hPa}$ decreases (Figure 11b). The maxima of divergence at $200 \mathrm{hPa}$ (Figure 11e) and geopotential height low (Figure 12b) both shift northeastward to the Yellow Sea and recede. The southwest winds (Figure 12b) are not so strong as the genesis period either. We can see that although the influences of MSLP lows seem to enlarge, backgrounds like the water vapor, divergence, and geopotential height which are essential to developments of Jianghuai cyclones fall into a substantial decline.

At the lysis time, it can be found that the wet tongue over the Yangtze-Huai River basin has vanished (Figure 9c). MSLP lows over the study area weaken obviously (Figure 10c). Convergence at $850 \mathrm{hPa}$ reduces and retreats to the south (Figure 11c), as well as the divergence at $200 \mathrm{hPa}$ (Figure 11f). It implies that the updraft decreases. The largest divergence center at $200 \mathrm{hPa}$ is located around the Korean Peninsula. As shown in Figure 12c, the geopotential height low and trough over Anhui and Jiangsu Provinces do not exist, as opposed to the genesis time (Figure 12a). The southwesterly winds are also weaker and only occupy the south (Figure 12c). Therefore, water vapor conditions, MSLP low, ascending flow, and baroclinicity all decrease, which is not supportive for the further developments of Jianghuai cyclones at the decaying stage.

\section{Summary}

Jianghuai cyclones are detected and tracked by an automatic tracking algorithm from 1979 to 2010 using the ERA-Interim reanalysis data. The climatological characteristics of Jianghuai cyclones are investigated. There is an obvious year-to-year variability in the numbers of Jianghuai cyclones, however, no significant trend can be found. Jianghuai cyclones are most frequent in spring. The numbers of Jianghuai cyclones are the highest in May. But in winter, Jianghuai cyclones are the fewest and inactive. The potential reasons are discussed. Jianghuai cyclones are formed in the lake area and in the lee of the Dabie Mountain area. The average deepening pressure of Jianghuai cyclones is mainly between 0 and $1 \mathrm{hPa}$ per $6 \mathrm{~h}$ and the maximum deepening pressure is between 0 and $2 \mathrm{hPa}$ per $6 \mathrm{~h}$. Generally speaking, Jianghuai cyclones are not too strong and mostly do not develop explosively. Therefore, the lifetime of Jianghuai cyclone is short (1-2 days). The main reason is that when Jianghuai cyclones move out from the origin areas, the water vapor is insufficient and baroclinicity is not strong, which cannot sustain them. In addition, the large-scale circulations at the formation, climax, and dissipation periods of Jianghuai cyclones are studied in this work. Wet tongue, MSLP low, and ascending flow avail the genesis of a Jianghuai cyclone. They provide favorable baroclinic situations, that is, warm and moist air taken up by the stronger southwesterly winds encountering the cold air from the north. A field which needs to be explored in further detail in the future, is the mechanism of formation and development of Jianghuai cyclones.

Author Contributions: L.X. wrote the paper and designed the numerical experiments; Y.Z. performed the experiments and drew all the figures; both two authors analyzed the results.

Funding: This study is supported by Foundation of Key Laboratory for Semi-Arid Climate Change of the Ministry of Education in Lanzhou University from the Fundamental Research Funds for the Central Universities (lzujbky-2018-kb02).

Acknowledgments: The authors appreciate the European Center for Medium-Range Weather Forecasts (ECMWF) to provide the ERA-Interim reanalysis data free. We thank the journal editors and the Special Issue editors for their helpful work. We also appreciate two anonymous reviewers and Frauke Feser for their constructive comments and suggestions. This work is contributed to the National Natural Science Foundation of China (41565002), and the program for Innovative Research Team in Science and Technology in University of Yunnan Province.

Conflicts of Interest: The authors declare no conflict of interest. 


\section{References}

1. Zheng, M.; Chang, E.K.; Colle, B.A. Ensemble sensitivity tools for assessing extratropical cyclone intensity and track predictability. Weather Forecast. 2013, 28, 1133-1156. [CrossRef]

2. Colle, B.A.; Booth, J.F.; Chang, E.K. A review of historical and future changes of extratropical cyclones and associated impacts along the US East Coast. Curr. Clim. Chang. Rep. 2015, 1, 125-143. [CrossRef]

3. Xu, X.N.; Jiao, P.J. The structure of Jianghuai cyclone in summer. J. Meteorol. Sci. 1984, 4, 189-196. (In Chinese)

4. Zhang, R.X.; Wang, Z.M. The structure features of Jianghuai cyclone. J. Meteorol. Sci. 1988, 8, 75-82. (In Chinese)

5. Hou, D.C. A comparative study on the structures of summer cyclones over the Changiiang-Huaihe valley. J. Meteorol. Sci. 1992, 12, 93-99. (In Chinese)

6. Wei, J.S.; Liu, J.Y.; Sun, Y.; Xu, Y.C. Climate characteristics of Jianghuai cyclone. J. Meteorol. Sci. 2013, 33, 196-201. (In Chinese)

7. Wang, Y.L.; Guan, Z.Y.; Jin, D.C.; Ke, D. Climatic characteristics and interannual (interdecadal) variations of cyclones over Changjiang-Huaihe River basin during late spring and early summer from 1980 to 2012. Trans. Atmos. Sci. 2015, 38, 354-361. (In Chinese)

8. Murray, R.J.; Simmonds, I. A numerical scheme for tracking cyclone centres from digital data Part I: Development and operation of the scheme. Aust. Meteorol. Mag. 1991, 39, 155-166.

9. Hodges, K.I. A general method for tracking analysis and its application to meteorological data. Mon. Weather Rev. 1994, 122, 2573-2586. [CrossRef]

10. Hodges, K.I. Feature tracking on the unit sphere. Mon. Weather Rev. 1995, 123, 3458-3465. [CrossRef]

11. Blender, R.; Fraedrich, K.; Lunkeit, F. Identification of cyclone-track regimes in the North Atlantic. Q. J. R. Meteorol. Soc. 1997, 123, 727-741. [CrossRef]

12. Rudeva, I.; Gulev, S.K. Climatology of cyclone size characteristic and their changes during the cyclone life cycle. Mon. Weather Rev. 2007, 135, 2568-2587. [CrossRef]

13. Zahn, M.; von Storch, H. Tracking polar lows in CLM. Meteorol. Z. 2008, 17, 445-453. [CrossRef] [PubMed]

14. Xia, L.; von Storch, H.; Feser, F. Quasi-stationarity of centennial Northern Hemisphere midlatitude winter storm tracks. Clim. Dyn. 2013, 41, 901-916. [CrossRef]

15. Xia, L.; von Storch, H.; Feser, F.; Wu, J. A study of quasi-millennial extratropical winter cyclone activity over the Southern Hemisphere. Clim. Dyn. 2016, 47, 2121-2138. [CrossRef]

16. Dee, D.P.; Uppala, S.M.; Simmons, A.J.; Berrisford, P.; Poli, P.; Kobayashi, S.; Andrae, U.; Balmaseda, M.A.; Balsamo, G.; Bauer, D.P.; et al. The ERA-Interim reanalysis: Configuration and performance of the data assimilation system. Q. J. R. Meteorol. Soc. 2011, 137, 553-597. [CrossRef]

17. Berrisford, P.; Dee, D.; Fielding, K.; Fuentes, M.; Kallberg, P.; Kobayashi, S.; Uppala, S.; Simmons, A. The ERA-Interim Archive Version 2.0; European Centre for Medium-Range Weather Forecasts: Reading, UK, 2011; pp. 1-16.

18. Gao, L.; Hao, L. Verification of ERA-Interim reanalysis data over China. J. Subtrop. Resour. Environ. 2014, 9, 75-81. (In Chinese)

19. Gao, Z.G.; Luo, J.X.; Liu, K.X. Evaluation of ERA-Interim Reanalysis data along coast of China. Mar. Sci. 2015, 39, 92-105. (In Chinese)

20. The Forecast Task Group of Jiangsu Provincial Weather Bureau. The Analysis and Forecasting of Important Weather in Jiangsu Province; Meteorological Press: Beijing, China, 1988; pp. 1-20. (In Chinese)

21. Wu, B.; Liu, C. The statistical investigation of the activity of extratropical cyclones in East Asia. J. Nanjing Univ. 1958, 2, 1-22. (In Chinese)

22. Xia, L.; Zahn, M.; Hodges, K.I.; Feser, F.; Storch, H. A comparison of two identification and tracking methods for polar lows. Tellus A 2012, 64. [CrossRef]

23. Sun, H.C.; Wei, J.S.; Li, C. Application of an objective discriminating method in the processes of two Jianghuai cyclones. Trans. Atmos. Sci. 2015, 38, 46-54. (In Chinese)

24. Qin, T.; Wei, L.X. The statistic and variance of cyclones entering coastal waters of China in 1979-2012. Acta Oceanol. Sin. 2015, 37, 43-52. (In Chinese)

25. Liu, J.Y. Study on Climatic Characteristics of Jianghuai Cyclone. Master's Thesis, Nanjing University of Information Science and Technology, Nanjing, China, 2012. (In Chinese) 
26. Hoskins, B.J.; McIntyre, M.E.; Robertson, A.W. On the use and significance of isentropic potential vorticity maps. Q. J. R. Meteorol. Soc. 1985, 111, 877-946. [CrossRef]

27. Uccellini, L.W. Process Contributing to the Rapid Development of Extratropical Cyclones; American Meteorological Society: Boston, MA, USA, 1990; pp. 81-106.

28. Gulev, S.K.; Zolina, O.; Grigoriev, S. Extratropical cyclone variability in the Northern Hemisphere winter from the NCEP/NCAR reanalysis data. Clim. Dyn. 2001, 17, 795-809. [CrossRef]

29. Hoskins, B.J.; Hodges, K.I. New Perspectives on the Northern Hemisphere Winter Storm Tracks. J. Atmos. Sci. 2002, 59, 1041-1061. [CrossRef]

30. Feser, F.; von Storch, H. A spatial two-dimensional discrete filter for limited-area-model evaluation purposes. Mon. Weather Rev. 2005, 133, 1774-1786. [CrossRef]

(C) 2018 by the authors. Licensee MDPI, Basel, Switzerland. This article is an open access article distributed under the terms and conditions of the Creative Commons Attribution (CC BY) license (http:/ / creativecommons.org/licenses/by/4.0/). 